\title{
BMJ Open Quantifying alcohol-related emergency admissions in a UK tertiary referral hospital: a cross-sectional study of chronic alcohol dependency and acute alcohol intoxication
}

To cite: Vardy J, Keliher T, Fisher J, et al. Quantifying alcohol-related emergency admissions in a UK tertiary referral hospital: a crosssectional study of chronic alcohol dependency and acute alcohol intoxication. BMJ Open 2016;6:e010005. doi:10.1136/bmjopen-2015010005

- Prepublication history and additional material is available. To view please visit the journal (http://dx.doi.org/ 10.1136/bmjopen-2015010005).

Received 3 November 2015 Revised 20 April 2016 Accepted 18 May 2016

\section{CrossMark}

\footnotetext{
${ }^{1}$ Emergency Department, Wishaw General Hospital, Wishaw, UK

2Emergency Department, Glasgow Royal Infirmary, Glasgow, UK

${ }^{3}$ ICU Department, Glasgow Royal Infirmary, Glasgow, UK ${ }^{4}$ Emergency Department, Hairmyres Hospital, East Kilbride, UK
}

Correspondence to Dr J Vardy; jennifer.vardy@lanarkshire. scot.nhs.uk

\section{ABSTRACT}

Objectives: Alcohol is responsible for a proportion of emergency admissions to hospital, with acute alcohol intoxication and chronic alcohol dependency (CAD) implicated. This study aims to quantify the proportion of hospital admissions through our emergency department (ED) which were thought by the admitting doctor to be (largely or partially) a result of alcohol consumption.

Setting: ED of a UK tertiary referral hospital. Participants: All ED admissions occurring over 14 weeks from 1 September to 8 December 2012. Data obtained for 5497 of 5746 admissions (95.67\%).

Primary outcome measures: Proportion of emergency admissions related to alcohol as defined by the admitting ED clinician.

Secondary outcome measures: Proportion of emergency admissions due to alcohol diagnosed with acute alcohol intoxication or CAD according to ICD-10 criteria.

Results: $1152(21.0 \%, 95 \% \mathrm{Cl} 19.9 \%$ to $22.0 \%)$ of emergency admissions were thought to be due to alcohol. $74.6 \%$ of patients admitted due to alcohol had $\mathrm{CAD}$, and significantly greater than the $26.4 \%$ with 'Severe' or 'Very Severe' acute alcohol intoxication $(p<0.001)$. Admissions due to alcohol differed to admissions not due to alcohol being on average younger (45 vs 56 years, $p<0.001$ ) more often male ( $73.4 \%$ vs $45.1 \%$ males, $p<0.001)$ and more likely to have a diagnosis synonymous with alcohol or related to recreational drug use, pancreatitis, deliberate selfharm, head injury, gastritis, suicidal ideation, upper gastrointestinal bleeds or seizures $(p<0.001)$. An increase in admissions due to alcohol on Saturdays reflects a surge in admissions with acute alcohol intoxication above the weekly average $(p=0.003)$. Conclusions: Alcohol was thought to be implicated in $21 \%$ of emergency admissions in this cohort. CAD is responsible for a significantly greater proportion of admissions due to alcohol than acute intoxication. Interventions designed to reduce alcohol-related admissions must incorporate measures to tackle CAD.

\section{Strengths and limitations of this study}

Data were collected by the treating clinician at the time of the admission decision thus obtaining the most accurate data on the influence of alcohol on the admission and the degree of acute intoxication at that precise time.

- A high inclusion rate of $95.67 \%$ reinforced by data showing little difference between omitted and included admissions indicates a low probability of selection bias and a high internal validity for this study.

- This study looked only at admissions and did not calculate the total burden of alcohol on the emergency department as no consideration was made of alcohol-related attendances that do not require admission but nonetheless use time and resources.

- Data on children aged under 13 years are probably incomplete as it is a protocol for ambulances and common practice for parents to present directly to the nearby paediatric hospital.

\section{INTRODUCTION}

Chronic alcohol dependency (CAD) and acute alcohol intoxication are both associated with alcohol-related hospital admissions in Scotland. ${ }^{12}$ Glasgow Royal Infirmary sees 5-6\% of all Scottish Emergency Department (ED) attendances but serves a deprived catchment with a higher prevalence of alcohol-related morbidity than the national average. ${ }^{3}{ }^{4}$ Alcohol is arguably the most harmful of all drugs to society as a whole $^{5}$ with alcohol-related hospital admissions one proxy for morbidity. Information Services Division (ISD) Scotland calculates that $91.2 \%$ of patients discharged with an alcohol-related diagnosis originate from emergency admissions. ${ }^{6}$ This puts the ED in 
prime position to gather information on alcohol-related admissions.

The primary aim of this study was to quantify the proportion of emergency admissions that are due to alcohol. Obtaining an accurate prevalence is useful for planning purposes in the immediate term and also as a baseline against which a repeat study could assess the effectiveness of alcohol-related harm reduction programmes.

One example of a potential intervention aimed at decreasing alcohol-related harm is the proposed introduction of a minimum price per unit alcohol, a measure shown to be effective at decreasing alcohol-related morbidity and mortality when implemented overseas. ${ }^{7-9}$ The Scottish government has passed legislation proposing a minimum unit price of 50 pence per unit of alcohol which epidemiological modelling predicts will decrease alcohol-related admissions by 6500 per annum by year $10 .{ }^{10} 11$ This study is powered to enable a future repeat study to detect a change in this order should one occur at this centre.

The secondary aim of this study was to analyse specific characteristics of patients admitted due to alcohol. We recorded the age, gender and diagnosis for all admissions and examined in more detail the relationship between acute alcohol intoxication, CAD and admission due to alcohol.

\section{METHODS}

A prospective, cross-sectional analysis undertaken within the ED of Glasgow Royal Infirmary, a city centre tertiary referral hospital. All emergency admissions were included over a 14-week period from 1 September to 8 December 2012.

Data were collected at the time the decision was made to admit by the clinician responsible for assessing and treating each admitted patient. This clinician could be an ED consultant, middle grade, junior (FY2 and above) or locum all of whom work throughout the department or an emergency nurse practitioner in the minor injuries area only. The data collection sheet used is included in table 1 , which contains an explanation of the definitions used.

Each day an admissions list was generated from departmental software to identify any missed admissions from the previous 24 hours. This enabled researchers to prompt clinicians to complete forms for any omitted patients.

\section{Definition of acute alcohol intoxication and CAD}

Acute alcohol intoxication and $\mathrm{CAD}$ were defined according to the International Classification of Diseases (ICD-10) Y91 classification system. ${ }^{12}$ ICD-10 Y91 contains objective criteria validated by the WHO for clinical assessment of alcohol intoxication. ${ }^{13}$ It classifies intoxication into 'Not', 'Mild', 'Moderate', 'Severe' and 'Very Severe'. Definitions for these are provided in online supplementary figure S1.

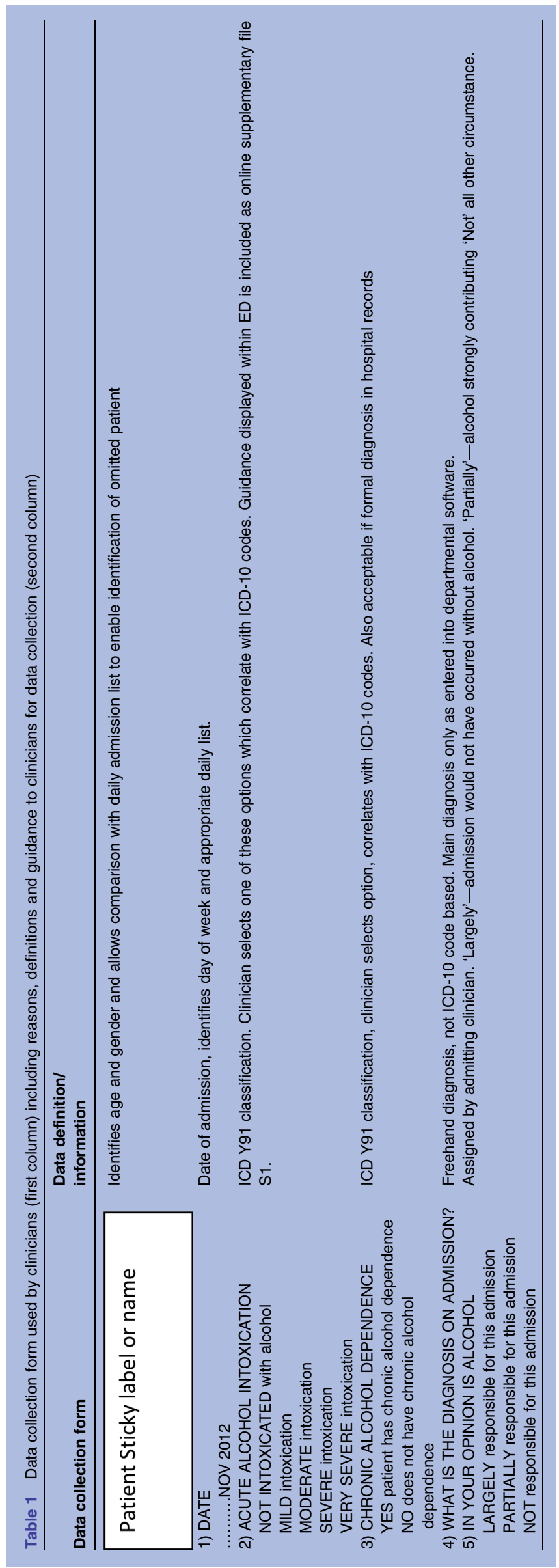


ED clinicians have been proved to successfully distinguish patients who are 'Not intoxicated' from those who are 'Mild' or 'Moderately' intoxicated and these from patients who are 'Severely' or 'Very Severely' intoxicated but are proved to be poor at distinguishing within these sub-categories. ${ }^{13}$ For this reason, pooled analysis of these three sub-categories was planned.

The definition of 'Severe' intoxication includes '...gross unsteadiness, severe difficulty in coordination, irrational behaviour, severely impaired judgement...'. Although 'Severe' intoxication by no means mandates admission, it is unlikely that patients with lesser degrees of intoxication would be admitted due to intoxication alone. Therefore, levels of 'Severe' or 'Very Severe' intoxicated were considered most likely to influence the admission decision.

CAD could be diagnosed either according to the definition given in ICD-10 Y91 ${ }^{12}$ or whether the diagnosis of CAD was formally recorded in the patient's hospital records.

Clinicians received verbal face-to-face training in the use of the ICD-10 classification system with copies displayed for reference within ED (see online supplementary figure S1). Researchers were all clinical colleagues within the department and remained available throughout the study period to answer questions as required.

\section{Definition of admission due to alcohol}

Admissions were categorised as 'Largely', 'Partially' and 'Not' due to alcohol by the admitting clinician. To minimise subjectivity, clinicians were given guidance to indicate 'Largely' if an admission would not have occurred without alcohol, 'Partially' when alcohol was a strongly contributing factor and 'Not' in all other circumstances. When results are reported as 'Due' or 'Not due' to alcohol, the 'Due' cohort includes admissions 'Largely' or 'Partially' due to alcohol.

\section{Statistical analysis and Power calculation}

Data were analysed using Minitab V.14. Continuous variables (such as age) were compared using the two sample t-test. Categorical variables (eg, gender) were assessed with the test of two proportions or by $\chi^{2}$ test when there was more than one degree of freedom (eg, degree of intoxication).

The sample size was calculated to allow a potential future study of identical size to be powered to detect a change in the proportion of admissions 'Due' to alcohol of $10 \%$ with Power V.0.8 and $\alpha=0.05$. This corresponds to the decrease in alcohol-related admissions by year 10 of minimum unit pricing predicted by mathematical modelling. ${ }^{11}$

\section{RESULTS}

There were 5746 admissions during the study period with data obtained for 5497 (95.67\%). Of these, 1152 admissions (21.0\%) were 'Due' to alcohol (95\% CI $19.9 \%$ to $22.0 \%$ ). This includes 785 admissions (14.3\%)
'Largely' due to alcohol and 367 admissions (6.7\%) 'Partially' due to alcohol.

Age

Figure 1 displays the ages of all 5497 emergency admissions indicating the number of patients in each age group admitted 'Due' or 'Not due' to alcohol.

Admissions 'Not due' to alcohol range in age from 0 to 100 years with a mean age of 56.5 years with numbers increasing through adulthood and peaking in the elderly.

Admissions 'Due' to alcohol range in age from 13 to 88 years with a significantly lower mean age of 45.4 years $(p<0.001)$. The majority of admissions 'Due' to alcohol were among working age adults and accounted for $28.6 \%$ of all emergency hospital admissions aged 2064 years.

\section{Acute alcohol intoxication}

Nine hundred and thirteen patients were acutely intoxicated with alcohol to some degree representing $16.6 \%$ of all admissions from ED. Intoxication was 'Mild' in 285 admitted patients, 'Moderate' in 323, 'Severe' in 215 and 'Very Severe' in 90 admissions.

The degree of intoxication correlates with the probability of admission 'Due' to alcohol. Only $6.5 \%$ of admissions of patients 'Not' intoxicated with alcohol were considered to be 'Due' to alcohol. This increased to $85.6 \%$ for 'Mild', $95.4 \%$ for 'Moderate', $99.5 \%$ for 'Severe' and $100 \%$ for 'Very Severe' intoxication $(\mathrm{p}<0.001)$.

In total, 305 admissions (5.5\% of all admissions) were 'Severely' or 'Very Severely' intoxicated; of whom, 304 admissions were 'Due' to alcohol. Thus acute alcohol intoxication was implicated in $26.4 \%$ of admissions 'Due' to alcohol.

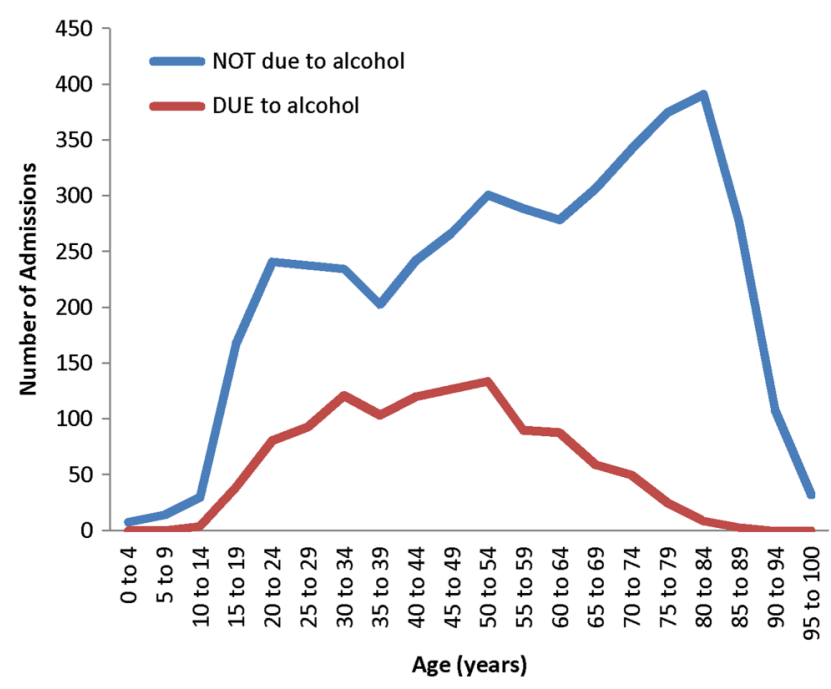

Figure 1 Ages of all emergency admissions 'Due' and 'Not' due to alcohol. 


\section{Chronic alcohol dependency}

Nine hundred and fifty-six admissions had a diagnosis of CAD. Of these, 860 were admitted due to alcohol. Thus, CAD was a factor in $74.6 \%$ of admissions 'Due' to alcohol.

The prevalence of CAD correlates with the degree of acute alcohol intoxication. 'Not' intoxicated admissions had a $7.1 \%$ prevalence of $\mathrm{CAD}$, rising to $63.2 \%$ for 'Mild', $67.5 \%$ for 'Moderate', $74.4 \%$ for 'Severe' and $78.8 \%$ for 'Very Severely' intoxicated admissions $(\mathrm{p}<0.001)$.

Among admissions with 'Severe' or 'Very Severe' intoxication, $75.1 \%$ had CAD. In contrast, only $24.1 \%$ of admissions with CAD were 'Severely' or 'Very Severely' intoxicated. Thus, the majority of patients with CAD admitted 'Due' to alcohol were not significantly intoxicated at the time of admission but instead admitted as a result of an alternate complication of alcohol misuse or trauma.

\section{Gender}

Among all 5497 emergency admissions, there were 2807 males $(51.1 \%, 95 \%$ CI $49.7 \%$ to $52.4 \%)$, giving a male: female ratio of 1.04. Among patients admitted 'Due' to alcohol, $73.4 \%$ were male (95\% CI $70.8 \%$ to $76.0 \%$ ),
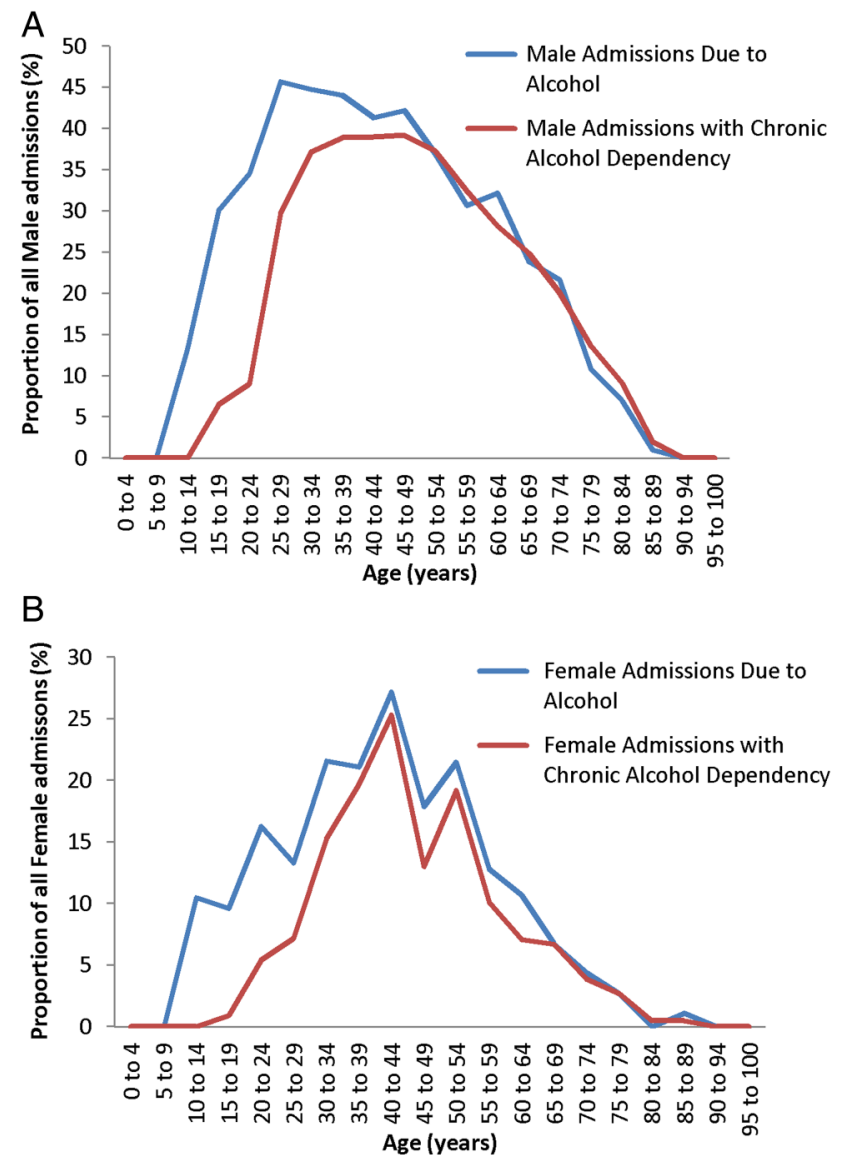

Figure 2 All male and female admissions indicating proportion 'Due' to alcohol or with CAD. with a significantly higher male:female ratio of 2.78 ( $\mathrm{p}=0.001$ ).

Male admissions were significantly more likely to be 'Severely' or 'Very Severely' intoxicated $(8.6 \%$ males vs $2.3 \%$ females, $\mathrm{p}<0.001)$ or to have CAD $(26.0 \%$ males vs $8.4 \%$ females $\mathrm{p}<0.001)$ than female admissions.

Figure 2 shows the proportion of all male (figure 2A) or all female (figure 2B) emergency hospital admissions that were 'Due' to alcohol or diagnosed with CAD by age group.

The proportion of emergency admissions 'Due' to alcohol peaks in men aged 25-29years; of whom, 45.7\% of admissions are 'Due' to alcohol. Prevalence remains high among men of working age with alcohol implicated in over $40 \%$ of emergency admissions of men aged 2549. The peak occurs later in women than that in men at the age of 40-44 years when with $27.2 \%$ of female admissions are 'Due' to alcohol.

CAD is commonly diagnosed in young men, including $29.8 \%$ of male admissions aged 25-29 years. Prevalence increases into middle age and peaks at $39 \%$ of men aged $40-44$ years. In total, over $35 \%$ of all male admissions aged 25-65 years had a diagnosis of CAD. Among women, CAD also peaks in the 40-44 years age group and present in $25.3 \%$ of all female admissions at this age.

For both men and women, there is a very strong correlation between the proportion of admissions 'Due' to alcohol and the prevalence of CAD in the middle aged and elderly. This correlation is not present among very young adults where the proportion of emergency admissions 'Due' to alcohol is greater than that with CAD.

\section{Relative influence of acute intoxication and CAD on admissions due to alcohol}

Figure 3 includes only the 1152 emergency admissions 'Due' to alcohol. It indicates the proportion of patients in each age group that were admitted 'Due' to alcohol and have a diagnosis of CAD or are classed as 'Severely' or 'Very Severely' intoxicated. Additional data by gender are provided in online supplementary figures S2 and S3.

Figure 3 demonstrates that the proportion of admissions 'Due' to alcohol with 'Severe' or 'Very Severe' intoxication shows little variation from the $26.4 \%$ mean between the ages of 20 and 74 years. In contrast, the proportion of admissions with a diagnosis of CAD increases rapidly with age being present in $35 \%$ of patients aged 20-24 years, $61.3 \%$ of patients aged 25-25 years and $73.9 \%$ of patients aged 30-34 years admitted 'Due' to alcohol. The proportion is high throughout middle age and the elderly with CAD present in $85 \%$ of admissions 'Due' to alcohol between 30 and 84 years of age.

\section{Diagnosis}

Figure 4 lists the primary admission diagnosis for all patients admitted 'Due' to alcohol. To be clear, it is the admission that is 'Due' to alcohol not the condition. 


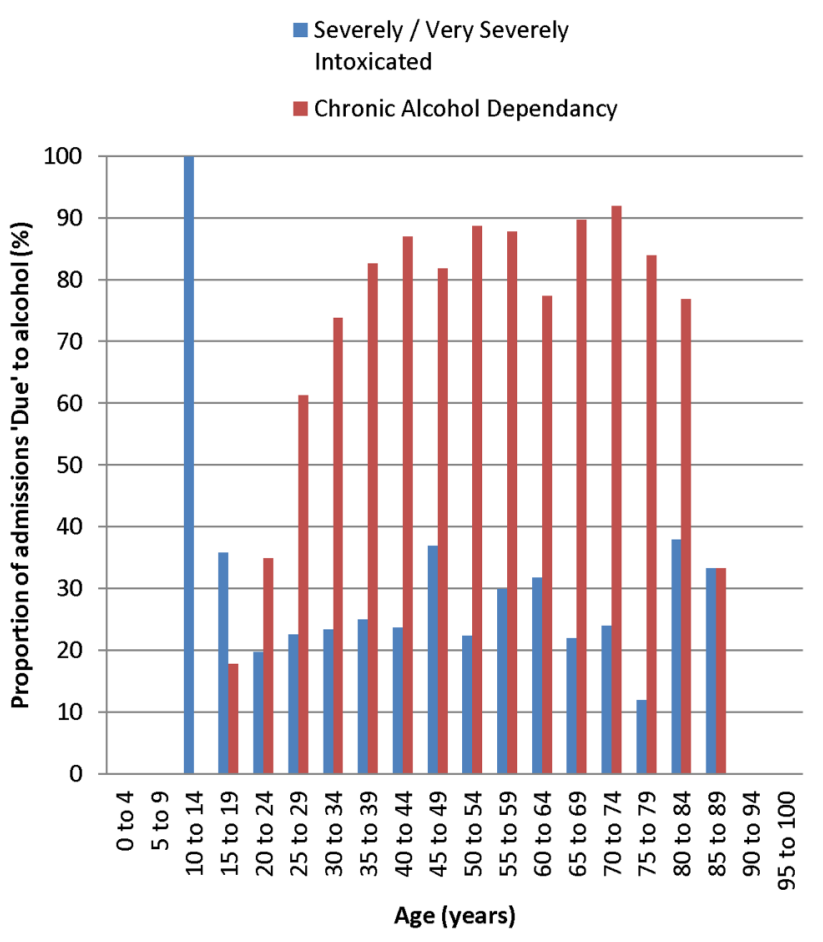

Figure 3 Proportion of admissions 'Due' to alcohol with CAD or 'Severe/Very Severe' intoxication.

For example $51.8 \%$ of patients admitted with a diagnosis of upper gastrointestinal bleed (UGIB) were admitted 'Due' to alcohol. This does not equate to $51.8 \%$ of UGIB being caused by alcohol.

Admissions 'Due' to alcohol are most strongly associated with conditions for which alcohol is synonymous, including alcoholic liver disease, alcohol intoxication, alcoholic ketoacidosis, alcohol withdrawal, Wernicke's encephalopathy and alcoholic hepatitis. Alcohol also has significant association with admissions due to complications of recreational drug use (alcohol implicated in $74.5 \%$ of admissions with this diagnosis), pancreatitis $(70.8 \%)$, deliberate self-harm/intentional overdose $(69.5 \%)$, head injuries $(68 \%)$, gastritis $(55.9 \%)$, suicidal ideation (55.6\%), upper GI bleeds (51.8\%) and seizures (48.1\%), all significant at $\mathrm{p}<0.001$.

Admissions for non-head trauma (defined as trauma to a part of the body other than the head), AF, falls, collapse and unexplained confusion were no more likely to be 'Due' or 'Not due' to alcohol. Acute coronary syndrome (ACS), hypoglycaemia/hyperglycaemia or other abdominal pain (abdominal pain other than pancreatitis or gastritis) had a small proportion of admissions associated with alcohol but were significantly more probable to be admitted 'Not due' to alcohol.

Numerically the most frequent admission diagnoses were not necessarily those most strongly associated with alcohol. $68 \%$ of the 306 head injury admissions were 'Due' to alcohol, a total of 208 admissions, making head injury the commonest diagnosis for admission 'Due' to alcohol. The next most prevalent numerically were deliberate self-harm or overdose (164 admissions 'Due' to alcohol), non-head trauma (113 admissions) and alcohol withdrawal (100 admissions).

Ninety admissions had a primary diagnosis of alcohol intoxication, considerably fewer than the 304 'Severely' or 'Very Severely' intoxicated patients admitted 'Due' to alcohol. This indicates that most significantly intoxicated patients had not intoxication but an alternative complication of alcohol misuse as an admission diagnosis.

\section{Days of the week}

Figure 5 shows the proportion of emergency admissions 'Due' to alcohol on each day of the week. The greatest proportion occurs on Saturdays when 28\% of admissions are 'Due' to alcohol, a value significantly higher than the weekly average of $21 \%(\mathrm{p}<0.001)$.

Figure 5 also displays the proportion of admissions diagnosed with CAD or with 'Severe' or 'Very Severe' intoxication each day. The proportion with $\mathrm{CAD}$ ranges from $14.8 \%$ on Sundays to $20.3 \%$ on Tuesdays and does not vary significantly throughout the week. The proportion with 'Severe' or 'Very Severe' intoxication ranges from $3.7 \%$ on Mondays to $8.7 \%$ on Saturdays. $8.7 \%$ is significantly higher than the $5.5 \%$ weekly average ( $p=0.003$ ) and contributes to the excess of admissions 'Due' to alcohol on Saturdays.

\section{Omitted patients}

Data were not obtained on 249 admissions (4.3\%), but we were able to extract age, gender and diagnosis from departmental software.

The reasons given for omissions included: patient seen by locums who could not be chased up $48 \%$, form filled by clinician but gone missing $17 \%$, unable to locate a regular staff member $8 \%$, form incomplete $8 \%$, clinician could not remember $3 \%$, no reason given $31 \%$.

Omitted and included patients did not differ significantly in terms of age ( 52.3 vs 54.2 years, $\mathrm{p}=0.186$ ) or gender (males $53.0 \%$ vs $51.1 \%, \mathrm{p}=0.547$ ). Nor did a predilection for any diagnosis exist $(\mathrm{p}=0.055-0.991)$ except for non-head injury trauma where omitted patients were over represented $(\mathrm{p}=0.002)$. This may be because locums, who were responsible for the majority of omissions, are commonly deployed in the minor injuries area of the ED where the majority of non-head trauma is seen.

\section{DISCUSSION}

\section{Principle findings}

$21.0 \%$ of emergency admissions were thought by the admitting ED clinician to be 'Due' to alcohol. Of these, alcohol was 'Largely' responsible for $14.3 \%$ of admissions and 'Partially' responsible for $6.7 \%$ of admissions.

CAD was present in $74.6 \%$ of emergency admissions 'Due' to alcohol. This is significantly higher than the $26.4 \%$ classed as 'Severely' or 'Very Severely' intoxicated. 
Figure 4 Admission diagnosis

'Due' to alcohol.

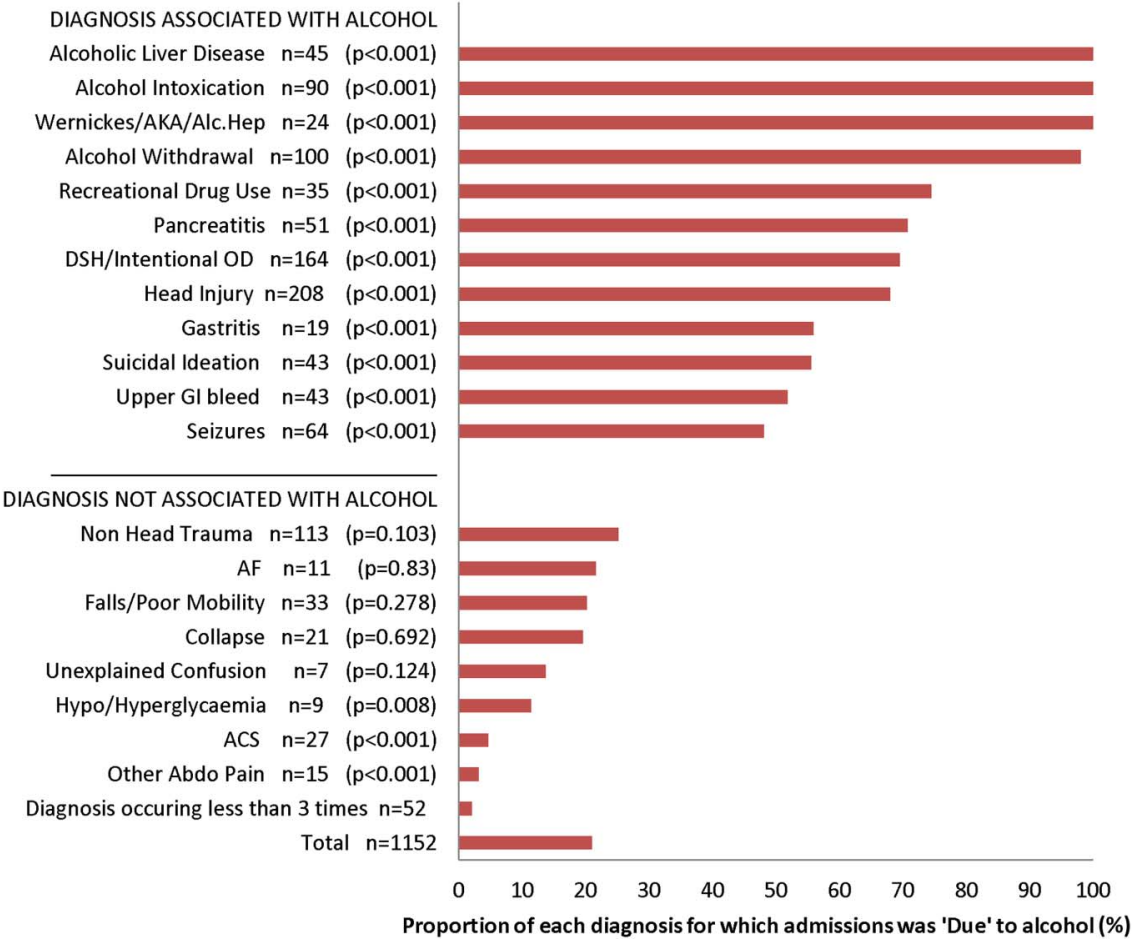

Admission diagnosis indicates that the majority of admissions due to alcohol were for complications of chronic alcohol misuse or trauma rather than acute intoxication.

Acute intoxication contributes to surges in admissions 'Due' to alcohol on Saturdays.

Alcohol harm reduction measures intended to decrease the number of hospital admissions attributed

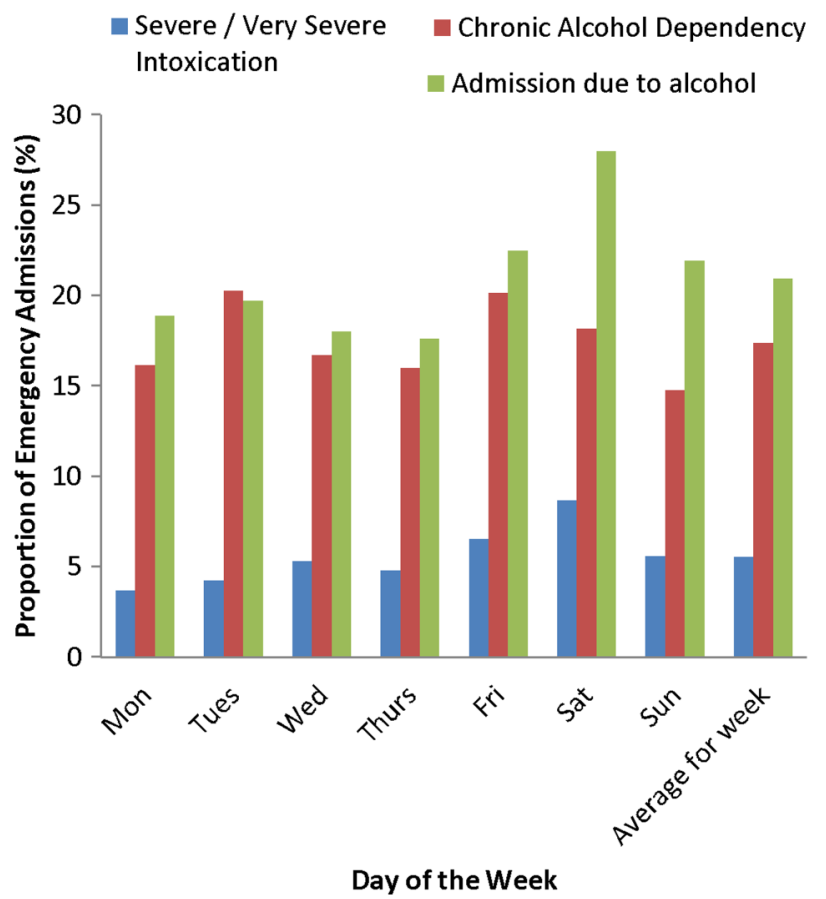

Figure 5 Admissions by day of the week, proportion 'Due' to alcohol, with CAD or 'Severe/Very Severe' intoxication. to alcohol will be most effective if directed towards reducing CAD.

\section{Strengths and limitations}

The main strength of this study is the timing and method of data collection. Data were collected by the clinician who treated the patient and made the decision to admit. This enabled an accurate first-hand assessment of the degree of acute alcohol intoxication at the time of admission and, more importantly, the overall influence of alcohol on the requirement for admission regardless of the clinical diagnosis or pre-existing comorbidities.

A potential source of bias is a reluctance to record a primary admission diagnosis of acute alcohol intoxication. It is understood that patients are not usually admitted with intoxication unless additional pathology is present or the patient is exceptionally vulnerable. This could affect the diagnosis list shown in figure 5 but would not influence the main findings of the study which were based on ICD-10 degrees of intoxication, not diagnosis.

This study looked at admissions only and should not be interpreted as in any way reporting the total burden of alcohol on the ED.

We also looked at admissions, not patients. One patient requiring multiple admissions cannot be differentiated from several different patients with one admission each.

\section{Comparison with other studies}

The majority of studies in the literature that examine alcohol-related admissions use a substantially different 
methodology based on coding of primary and secondary discharge diagnosis. In some circumstances, discharge coding captures more alcohol-related discharges than our methodology when patients with pre-existing alcohol-related comorbidities have alcohol implicated during an unrelated admission. For example, the Health and Social Care Information Centre (HSCIC) assigns an 'alcohol attributable fraction' to each code ${ }^{14}$ and classes an admission of a patient with breast or bowel cancer as 'partially' due to alcohol ${ }^{15}$ regardless of the reason for admission whereas an admission for a complication of these conditions would be classed as 'Not' due to alcohol in this study.

In other circumstances, discharge coding may underestimate the influence of alcohol on admissions by failing to consider factors such as social circumstances and patient safety which may influence the decision to admit. The degree of acute alcohol intoxication may also be assessed more accurately at the time of admission than by discharge coding.

The HSCIC in England implicates alcohol as the primary cause in $5.3 \%$ and a secondary cause in $26.8 \%$ of emergency admissions ${ }^{14}$ whereas the Information Service Division (ISD) calculates that alcohol is responsible for $6.1 \%$ of emergency admissions in Scotland. ${ }^{6} 16$ This study's $21.0 \%$ admission rate due to alcohol, or even the $14.3 \%$ 'Largely' due to alcohol, is higher than either ISD or HSCIC figures and probably represents a true excess of alcohol-related admissions to this centre.

This study detected a significantly larger proportion of admissions 'Due' to alcohol among men and young adults than that reported in the literature. This centre saw 2.78 male for each female admission 'Due' to alcohol, significantly higher than either the 2.37 male: female predominance calculated by ISD Scotland $(\mathrm{p}<0.001)$ or the 1.59 ratio reported by HSCIC. ${ }^{6}{ }^{17}$ In addition, a significantly higher proportion of admissions 'Due' to alcohol occurred in young adults aged 2049 years which at this centre accounted for $56.1 \%$ of all admissions 'Due' to alcohol. This is significantly higher than the proportion of patients aged 20-49 years reported by ISD $(45.6 \%, \mathrm{p}=0.038)$ and the proportion of patients of all admissions 'Due' to alcohol, aged 2554 years, calculated by HSCIC $(35.2 \%) .^{6} 17$

This study demonstrated a relationship between alcohol and specific admission diagnosis. An association between alcohol and diagnosis including injury or deliberate self-harm has been shown before, ${ }^{18-21}$ but in this study the admitting clinician stated whether alcohol was responsible for actual admission enabling this work to be better placed to infer causality.

\section{The meaning of the study and implications for clinicians and policymakers}

Alcohol is responsible for a considerably higher proportion of emergency admissions to this centre than the national average. $\mathrm{CAD}$ has a much greater influence on the number of admissions due to alcohol than acute alcohol intoxication and has a high prevalence in the local population particularly among young and middle aged men.

The aetiology of this high prevalence of CAD may be related to high levels of deprivation in the local catchment area. The UK Department of Work and Pensions recognises that the relationship between unemployment and alcohol misuse is at least partially causal ${ }^{22}$ and at the time of the study employment in Glasgow averaged only $65.2 \% .^{23}$

The greater influence of CAD over acute intoxication on numbers of emergency admissions has been demonstrated before ${ }^{17}$ but is more pronounced in this study because of the higher prevalence of $\mathrm{CAD}$ is this population.

Hospital admissions due to alcohol represent a surrogate marker for alcohol-related morbidity and mortality. The high prevalence of CAD, even among young adults, begs the meaningful contemplation of any plausible intervention that could reduce the burden of alcoholrelated harm. The most effective method to decrease alcohol-related emergency admissions will be to adopt measures targeted at decreasing the prevalence of CAD.

\section{Unanswered questions and future research}

This study considered alcohol-related admissions but did not examine patients with alcohol-related presentations who are treated and discharged from ED. These patients affect heavily on ED workload ${ }^{24}$ and quantifying the ED resources and staff time required to manage patients discharged from ED following an alcohol-related presentation would be of value. This would be possible using this study's methodology but would require much greater researcher input.

Legislation to introduce a minimum unit price has been passed by the Scottish government. The legislation has received much press coverage ${ }^{25}$ and an industry-led challenge currently lies with the Court of Session in Edinburgh. Minimum pricing is an effective public health measure capable of decreasing morbidity and mortality from acute and chronic sequelae of alcohol misuse of alcohol by decreasing population wide over consumption. ${ }^{7-9}$ A decrease in the number of alcohol-related admissions to hospital of 6500 per annum for Scotland by year 10 post implementation is one of the four targets derived from epidemiological modelling. Should minimum unit pricing be introduced, then a repeat cross-sectional study of the same size as this would be powered to detect a change in the proportion of hospital admissions due to alcohol of this magnitude at this centre, should one exist.

Acknowledgements Srinivasa Vittal Katikireddi-Medical Research Council/ Chief Scientists Office, Social and Public Health Sciences Unit, University of Glasgow.

Contributors JV and RC conceived the study methodology. JV, TK, FR, CB, FC, LBI, LBa, JF, EP, AC, RC, JF, MC collected data. JV, TK, FR, CB, FC, LBI, $\mathrm{LBa}$, JF, EP, AC, RC, JF, MC compiled data. JV and TK prepared the final draft.

All authors approved the final draft. 
Funding This research received no specific grant from any funding agency in the public, commercial or not-for-profit sectors.

Competing interests None declared.

Provenance and peer review Not commissioned; externally peer reviewed.

Data sharing statement No additional data are available.

Open Access This is an Open Access article distributed in accordance with the Creative Commons Attribution Non Commercial (CC BY-NC 4.0) license, which permits others to distribute, remix, adapt, build upon this work noncommercially, and license their derivative works on different terms, provided the original work is properly cited and the use is non-commercial. See: http:// creativecommons.org/licenses/by-nc/4.0/

\section{REFERENCES}

1. McDonald SA, Hutchinson SJ, Bird SM, et al. Association of self-reported alcohol use and hospitalization for an alcohol-related cause in Scotland: a record-linkage study of 23,183 individuals. Addiction 2009;104:593-602.

2. The Scottish Government website. The societal cost of alcohol misuse in Scotland for 2007. http://www.scotland.gov.uk/ Publications/2009/12/29122804/6

3. NHS Scotland Emergency Department Activity. http://www. isdscotland.org/Health-Topics/Emergency-Care/Publications/ 2012-05-29/ED-pub-4hr-2011-12.pdf

4. Scottish Index of Multiple Deprivation. http://www.scotland.gov.uk/ Resource/0041/00410731.pdf

5. Nutt D, King L, Phillips L. Drug harms in the UK: a multicriteria decision analysis. Lancet 2010;376:1558-65.

6. Information Services Division (ISD) Scotland. Alcohol related hospital statistics 2012/2013. Published 24/2/14. https://isdscotland. scot.nhs.uk/Health-Topics/Drugs-and-Alcohol-Misuse/Publications/ 2014-02-25/2014-02-25-ARHS2012-13-Report.pdf?13326662779

7. Stockwell T, Auld MC, Zhao J, et al. Does minimum pricing reduce alcohol consumption? The experience of a Canadian province. Addiction 2012;107:912-20.

8. Wagenaar AC, Tobler AL, Komro KA. Effects of alcohol tax and price policies on morbidity and mortality: a systematic review. Am J Public Health 2010;100:2270-8.

9. Zhao J, Stockwell T, Martin G, et al. The relationship between minimum alcohol prices, outlet densities and alcohol-attributable deaths in British Columbia, 2002-09. Addiction 2013;108:1059-69.

10. The Scottish government website. Minimum Pricing. http://www. scotland.gov.uk/Topics/Health/Services/Alcohol/minimum-pricing
11. Purshouse RC, Meier PS, Brennan A, et al. Estimated effect of alcohol pricing policies on health and health economic outcomes in England: an epidemiological model. Lancet 2010;375:1355-64.

12. The ICD-10 Classification of Mental and Behavioural Disorders. http://www.who.int/substance_abuse/terminology/ ICD10ClinicalDiagnosis.pd

13. WHO collaborative study on Alcohol and Injuries: Final Report. http:// www.who.int/substance_abuse/publications/alcohol_injuries_final_ report.pdf

14. Health and Social care Information Centre. Statistics on England 2013. Published 30/5/13. http://www.hscic.gov.uk/catalogue/ PUB10932

15. Health and Social Care Information Centre. Statistics on Alcohol: England. 2012. http://www.hscic.gov.uk

16. Information Services Division (ISD) Scotland. http://www.isdscotland org/Health-Topics/Hospital-Care/Inpatient-and-Day-Case-Activity/

17. Jones L, Bellis MA, Dedman D, et al. Alcohol attributable fractions for England. Alcohol attributable mortality and hospital admission. North West Public Health Observatory. http://www. alcohollearningcentre.org.uk/_library/AlcoholAttributableFractions.pdf

18. Borges G, Cherpitel CJ, Orozco R, et al. Acute alcohol use and the risk of non-fatal injury in sixteen countries. Addiction 2006;101:993-1002.

19. Cherpitel CJ, Ye Y, Watters K, et al. Risk of injury from alcohol and drug use in the emergency department: a case-crossover study. Drug Alcohol Rev 2012;31:431-8.

20. Zerhouni $\mathrm{O}$, Bègue L, Brousse $\mathrm{G}$, et al. Alcohol and violence in the emergency room: a review and perspectives from psychological and social sciences. Int $J$ Environ Res Public Health 2013;10:4584-606.

21. Ness J, Hawton $\mathrm{K}$, Bergen $\mathrm{H}$, et al. Alcohol use and misuse, self-harm and subsequent mortality: an epidemiological and longitudinal study from the multicentre study of self-harm in England. Emerg Med J 2015;32:793-9.

22. Bauld L, Carroll C, Hay G, et al. Alcohol misusers' experiences of employment and the benefits system. Department of Work and Pensions. https://www.gov.uk/government/uploads/system/uploads/ attachment_data/file/214493/rrep718.pdf

23. Rehm J, Greenfield TK, Rogers JD. Average volume of alcohol consumption, patterns of drinking, and all cause mortality. Results from the US National Alcohol survey. Am J Epidemiol 2001;153:64-71.

24. Pirmohamed M, Brown C, Owens L, et al. The burden of alcohol misuse on an inner-city general hospital. QJM 2000;93:291-5.

25. Hilton S, Wood K, Patterson C, et al. Implications for alcohol minimum unit pricing advocacy: what can we learn for public health from UK newsprint coverage of key claim-makers in the policy debate? Soc Sci Med 2014;102:157-64. 